\title{
Ursolic Acid Improves Liver Transplantation and Inhibits Apoptosis in Miniature Pigs Using Donation After Cardiac Death
}

\author{
Wei Zhou Li Lin $^{\mathrm{b}} \quad$ Ying Cheng ${ }^{\mathrm{a}} \quad$ Yongfeng Liu \\ aDepartment of Hepatobiliary Surgery, the First Affiliated Hospital of China Medical University, \\ Shenyang, 'Institute of Respiratory Disease, the First Affiliated Hospital of China Medical University, \\ Shenyang, China
}

\section{Key Words}

Ursolic acid $\bullet$ Liver transplantation $•$ Apoptosis $•$ Cardiac death

\begin{abstract}
Background: Ursolic acid (UA) possesses extensive pharmacological activities, including anti-oxidation, anti-infection, anti-inflammation, anti-tumor, liver protection. This study was designed to investigate the effect of UA on liver transplantation after liver transplantation using donation after cardiac death (DCD), and to assess the mechanisms. Methods: 24 healthy experimental pigs were randomly divided into control and experimental groups. Each group received six DCD liver transplantations. In the experimental group, the recipient pigs received $120 \mathrm{mg} / \mathrm{kg}$ UA $4 \mathrm{~h}$ before surgery by intraperitoneal injection. The liver tissues and vein blood were collected 0 h, 1 h, 3 h, 6 h, 12 h and 24 h after transplantation. Morphological change, malondialdehyde (MDA) level, protein kinase-like ER kinase (PERK)-CHOP signaling pathway and apoptosis in liver tissue and serum aminotransferase (ALT) level were assessed. Results: Compared with control group, ALT level was significantly decreased $(P<0.05)$ and pathological changes in liver were ameliorated in experimental group. UA treatment also decreased MDA level in liver tissue and attenuated the apoptosis. Compared with control group, Bax decreased and $\mathrm{BCl}-2$ increased in UA-treated group. Importantly, UA decreased p-PERK, PERK, p-eIF2 $\alpha$, eIF2 $\alpha$, ATF4 and CHOP levels compared with control group. Conclusion: Our results showed that UA treatment could improve the DCD liver transplantation likely through inhibiting apoptosis and PERK-CHOP pathway.
\end{abstract}

\section{Introduction}

Liver transplantation is the only effective treatment for end-stage liver disease, which brings therapeutic hopes to patients with end-stage liver diseases [1, 2]_ENREF_1_ENREF_1. However, insufficient donor restricts the development of liver transplantation. Donation 
after cardiac death (DCD) enriches the source of donor to some extent $[3,4]$. However, the organs experience a process of ischemia and hypoxia before cardiac arrest [5, 6]_ENREF_5 ENREF_1. Therefore, organ injury will aggravate and complications will increase after surgery. Severe ischemia / reperfusion injury (IRI) is a leading cause of poor prognosis of DCD liver transplantation [7]_ENREF_2. Therefore, a lot of methods were tried to improve the function of grafted DCD [8]. Current studies have indicated that endoplasmic reticulum stress (ERS) plays an important role in ischemia injury after DCD liver transplantation [9]_ ENREF_1, especially the protein kinase-like ER kinase (PERK) pathway [10]_ENREF_1.

External factors stimulate the accumulation of unfolded and incorrectly folded proteins in the endoplasmic reticulum, which will lead to ERS [11]_ENREF_3. The unfolded protein response (UPR) was stimulated in ERS. UPR initiates to decrease new protein synthesis, correct protein folding and modification by molecular chaperone, and promote the protein degradation through ubiquitin-proteasome system to rescue endoplasmic reticulum [12] ENREF_1. However, once the stimulus is too strong or persistent or surpasses the endurance capacity of ER, it will activate the ERS-related apoptosis [13, 14]_ENREF_12_ENREF_1.

The UPR was achieved by three transmembrane molecules--PERK, inositol-requiring enzyme $1 \alpha$ (IRE1 $\alpha$ ) and activating transcription factor 6 (ATF6). There was study implicating that PERK-eIF2 $\alpha$-ATF4-CHOP pathway was obviously activated in the rat liver transplantation model [10]_ENREF_1. Moreover, this signaling pathway regulates the ischemia reperfusion injury after DCD liver transplantation through apoptosis [10]_ENREF_1. Meanwhile, endoplasmic reticulum stress is also involved in diseases such as non-alcoholic fatty hepatitis, myocardial injury in myocardial infarction [15, 16]_ENREF_15_ENREF_1.

Ursolic acid (UA) belongs to pentacyclic triterpenes and widely exists in natural plants. The pharmacological activities of UA include anti-oxidation, anti-infection, anti-inflammation, anti-tumor, liver protection, etc $[15,16]$ ENREF_16_ENREF_1. Previously, UA was reported to down-regulate PERK-eIF2 $\alpha$-ATF4-CHOP pathway to reduce apoptosis [15]_ENREF_1. In this study, we aimed to investigate the effects of UA on the tissue damage after DCD liver transplantation and mechanisms involved.

\section{Materials and Methods}

\section{Animal model and treatment}

24 healthy adult Bama miniature pigs (12 months, weight 30-40 kg, half male and half female) were provided by Shenyang Agricultural University (China). As a reference, miniature pigs in both genders were applied in the transplantation experiments [17]. In our study, the pigs were randomly divided into two groups: control group and experimental group (12 in each group). Each group had 6 donors and 6 recipients and pigs in each pair weighed similarly. The pigs in both groups received 6 DCD orthotopic liver transplantations. DCD model was established using the method of asphyxia as previously described [18], that is, stopping oxygen supplement after anesthesia and muscle relaxation. The donors were anesthetized by pump controlled intravenous injection of propofol and inhalation of isoflurane, and we need to do tracheal intubation. During this period, the blood pressure was monitored by cephalic artery catheterization. Abdomen was opened and hepatic portal vein and infrarenal aorta abdominalis were isolated. Pancuronium was applied to relax the muscle. After that, the respirator was withdrawn. The donor pigs went into cardiac arrest. The time period from respirator withdrawing to circulatory arrest was about $19.25 \pm 3.4 \mathrm{~min}$, comparable between two groups. The standard of circulatory arrest is heart stoppage or arterial blood pressure lower than $25 \mathrm{mmHg}$ and pulse pressure lower than $20 \mathrm{mmHg}$. The cold perfusion was completed by using $4^{\circ} \mathrm{C}$ UW solution, respectively $1000 \mathrm{ml}$ through portal vein and aorta abdominalis. Orthotopic liver transplantation was conducted and blood flow was initiated after the portal vein and suprahepatic vena cava were anastomosed. In the two groups, anhepatic phase was about $41.7 \pm 2.8 \mathrm{~min}$. Blood loss was estimated to be 300-500 ml. After surgery, all the transplanted livers and recipients survived for at least 24 hours. $24 \mathrm{~h}$ after transplantation, the pigs were euthanized. In control group, the pigs received DCD orthotopic liver transplantation without intervention. In experimental group, 4 hours before the surgery, recipient pigs received $120 \mathrm{mg} / \mathrm{kg}$ UA by intraperitoneal injection. Selection of the dose of UA was referred 


\section{Cellular Physiology Cell Physiol Biochem 2017;43:331-338 and Biochemistry Published online: August 31, 2017 年 $\begin{aligned} & \text { O 2017 The Author(s). Published by S. Karger AG, Basel } \\ & \text { www.karger.com/cpb }\end{aligned}$

to the literature [19]. The liver tissues and venous blood were collected at $0 \mathrm{~h}$ (donor liver), and $1 \mathrm{~h}, 3 \mathrm{~h}$, $6 \mathrm{~h}, 12 \mathrm{~h}$ and $24 \mathrm{~h}$ after when the blood circulation of transplanted liver was established (anastomosis of suprahepatic vena cava and hepatic portal vein were completed and blood flow was opened).

The 0-h time point referred to the time immediately after laparotomy in donors. The 1-h time point after opening of blood flow was just before the abdomen was closed (we successively sewed up the infrahepatic vena cava, hepatic artery, and common bile duct). The liver tissue was also obtained at 3-h, 6-h, 12-h and 24-h after transplantation under anesthesia through the incision which was partially closed. All experimental procedures were approved by the Animal Care and Use Committee of the First Affiliated Hospital of China Medical University.

\section{Immunohistochemistry}

Immunostaining of histological sections was performed using monoclonal antibodies against Bax (1:200, Abcam, USA), Caspase 3 (1:200, Abcam, USA) and Bcl-2 (1:200, Abcam, USA). Endogenous peroxidase activity was blocked with $3 \%(\mathrm{vol} / \mathrm{vol}) \mathrm{H}_{2} \mathrm{O}_{2}$ for 5 minutes. After that, tissues were incubated with primary antibody overnight at $4{ }^{\circ} \mathrm{C}$ followed by a 30 -minute incubation with secondary antibody (Dako, Carpinteria, CA) and visualization with DAB chromagen for 3 minutes.

\section{Western blotting}

Protein was abstracted from liver tissue for western blotting as previously described [20]_ENREF_1. The antibodies, including anti-PERK, anti-p-PERK, anti-eIF2 $\alpha$, anti-p-eIF2 $\alpha$, anti-ATF 4 and anti-CHOP (Santa Cruz, USA) were incubated overnight at $4^{\circ} \mathrm{C}$. The ECL reagent kit was applied to assist the staining. The blots were scanned by ChemiDocTM XRS (Bio-Rad, USA). The grey density was analyzed by Image J 7.0 software.

\section{Biochemical detection}

Serum alanine aminotransferase (ALT) and liver malondialdehyde (MDA) were detected according to the assay kits (Beyotime, Ningbo, China).

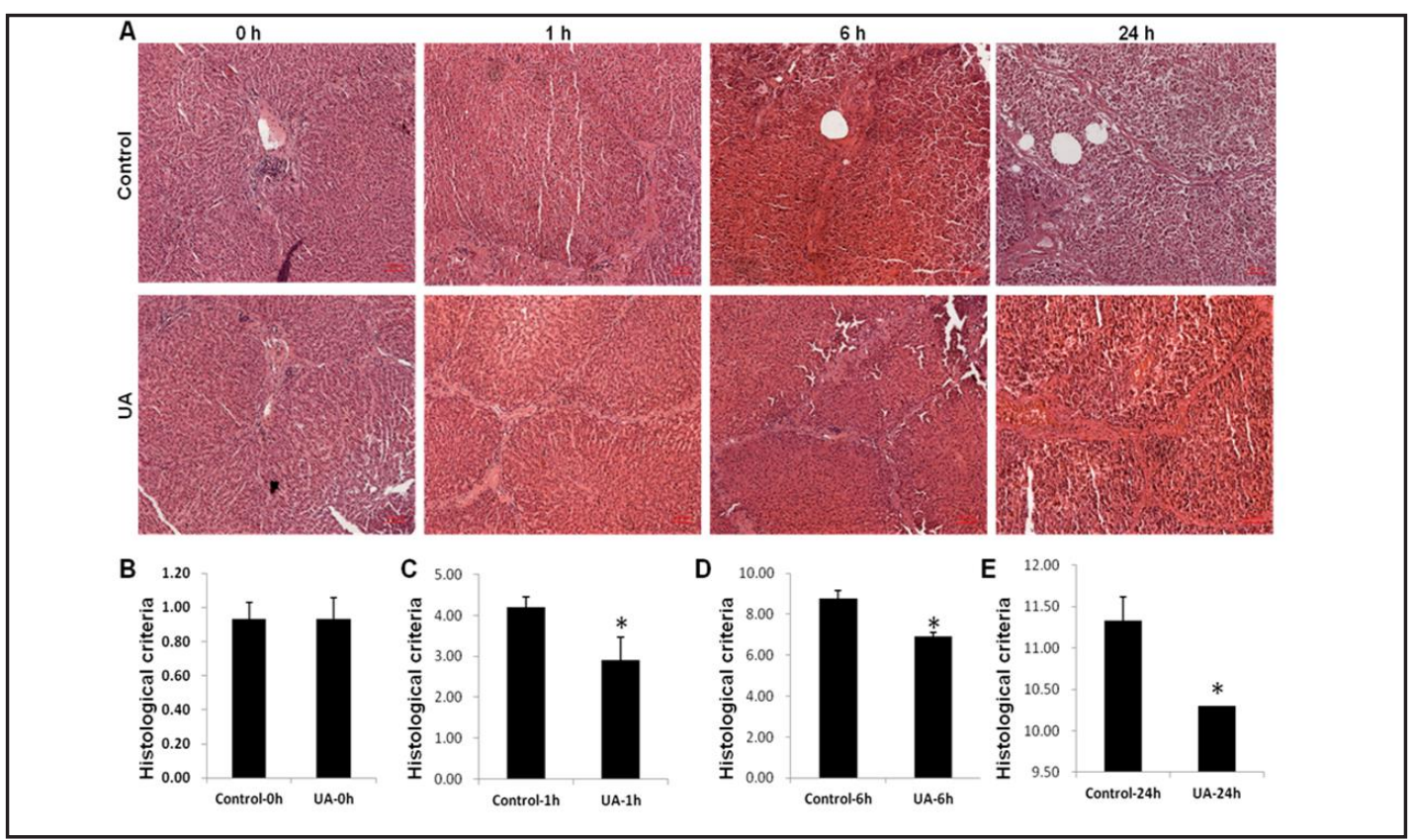

Fig. 1. HE staining of liver tissue. Representative images for $0 \mathrm{~h}, 1 \mathrm{~h}, 6 \mathrm{~h}$ and $24 \mathrm{~h}$ were obtained from control and UA groups (100X). B) Quantification data of the histological criteria at $0 \mathrm{~h}$. C) Quantification data of the histological criteria at $1 \mathrm{~h}$. D) Quantification data of the histological criteria at $6 \mathrm{~h}$. E) Quantification data of the histological criteria at $24 \mathrm{~h} .{ }^{*} \mathrm{P}<0.05$ compared with control group. 


\section{Cellular Physiology

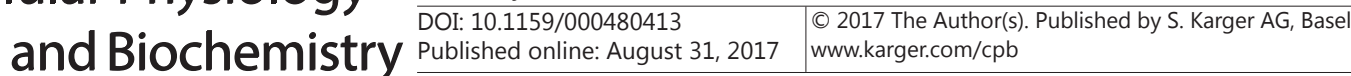

Tunel

Apoptosis was detected using Tunel assay according to the instruction of the assay kit (Beyotime, Ningbo, China).

\section{Statistical analyses}

Data were presented as means \pm standard deviations. One-way analysis of variance with posthoc Bonferroni test for multiple comparisons was performed. Differences were considered significant at $\mathrm{p}<0.05$.

\section{Results}

UA treatment improved DCD liver transplantation.

HE staining was applied to detect the morphological changes of the transplanted liver tissue. As shown in Fig.1, there were no morphological difference between control group and UA-treated group at the 0 -h time point. However, cell edema, liver sinus congestion, local necrosis, unclear cell boundary, infiltration of periportal lymphocyte gradually appeared in control group, especially at 24-hafter transplantation. Although these pathological changes were also observed in experimental group, UA obviously mitigated those abnormalities.

ALT level was also detected in our study. As shown in Fig.2, serum ALT level was significantly decreased in experimental group at $3 \mathrm{~h}, 6 \mathrm{~h}, 12 \mathrm{~h}$ and $24 \mathrm{~h}$ time points compared with control group.

Serum MDA level was also compared between two groups. As shown in Fig.3, significant difference was observed between the two groups at $12 \mathrm{~h}$ and $24 \mathrm{~h}$ after transplantation.

UA treatment inhibited apoptosis of the transplanted liver

We compared the apoptosis between control and experimental groups. As shown in Fig.4, apoptotic cells appeared in control group at the 3 -h and aggravated at $6-\mathrm{h}$ time point. In UA treated group, although apoptosis was still found, the level was decreased obviously compared with control group.

The apoptosis-regulators were also compared (Fig.5). Compared with control,

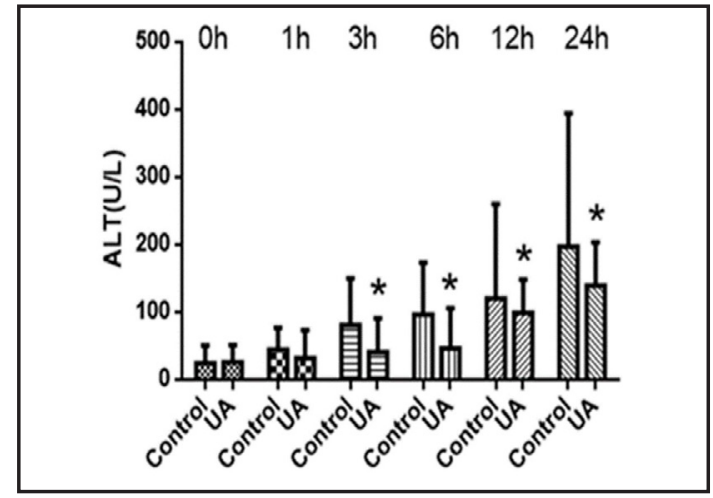

Fig. 2. Change of ALT level after UA treatment. Data were presented with mean and SD. ${ }^{*} \mathrm{P}<0.05$ compared with control group

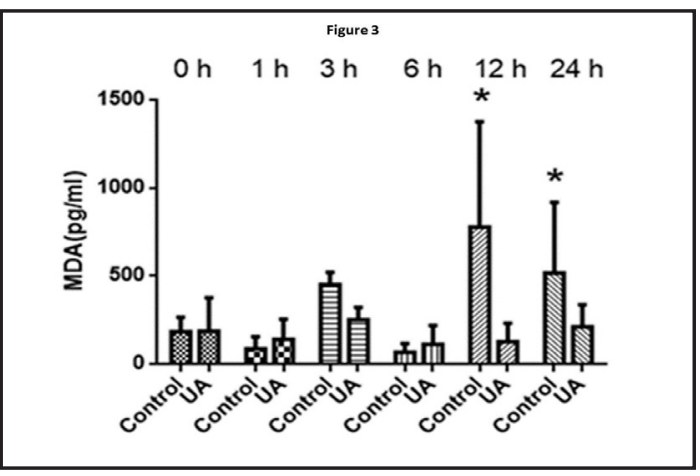

Fig. 3. Change of MDA level after UA treatment. Data were presented with mean and SD. ${ }^{*} \mathrm{P}<0.05$ compared with control group.

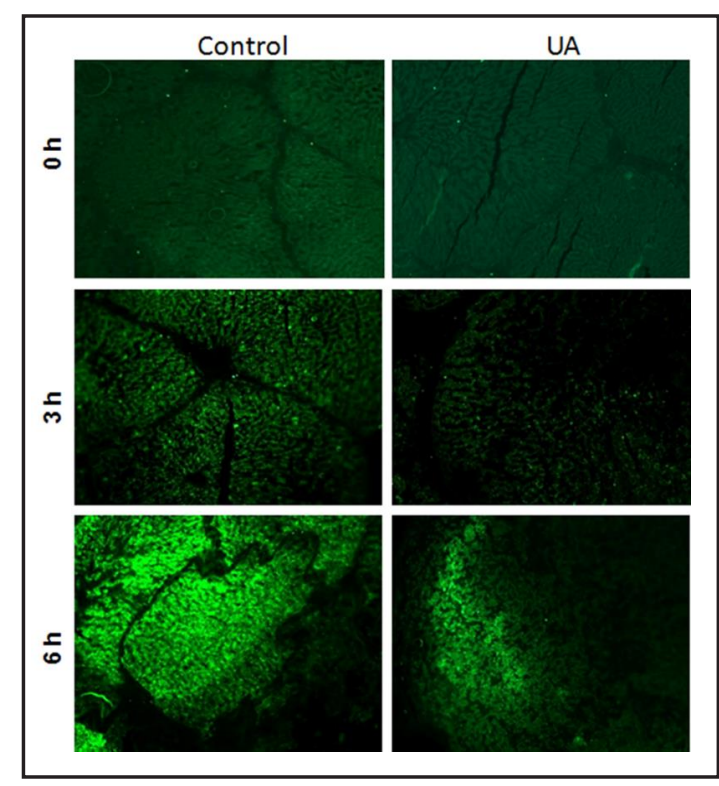

Fig. 4. Apoptosis detected by Tunel assay. 
Fig. 5. Expression of apoptosis related factors after $6 \mathrm{~h}$ after UA treatment. A) Representative images of Caspase-3, Bcl-2 and Bax. B) Quantification data of Caspase-3. C) Quantification data of Bax. D) Quantification data of Bcl-2. $* \mathrm{P}<0.05$ compared with control.
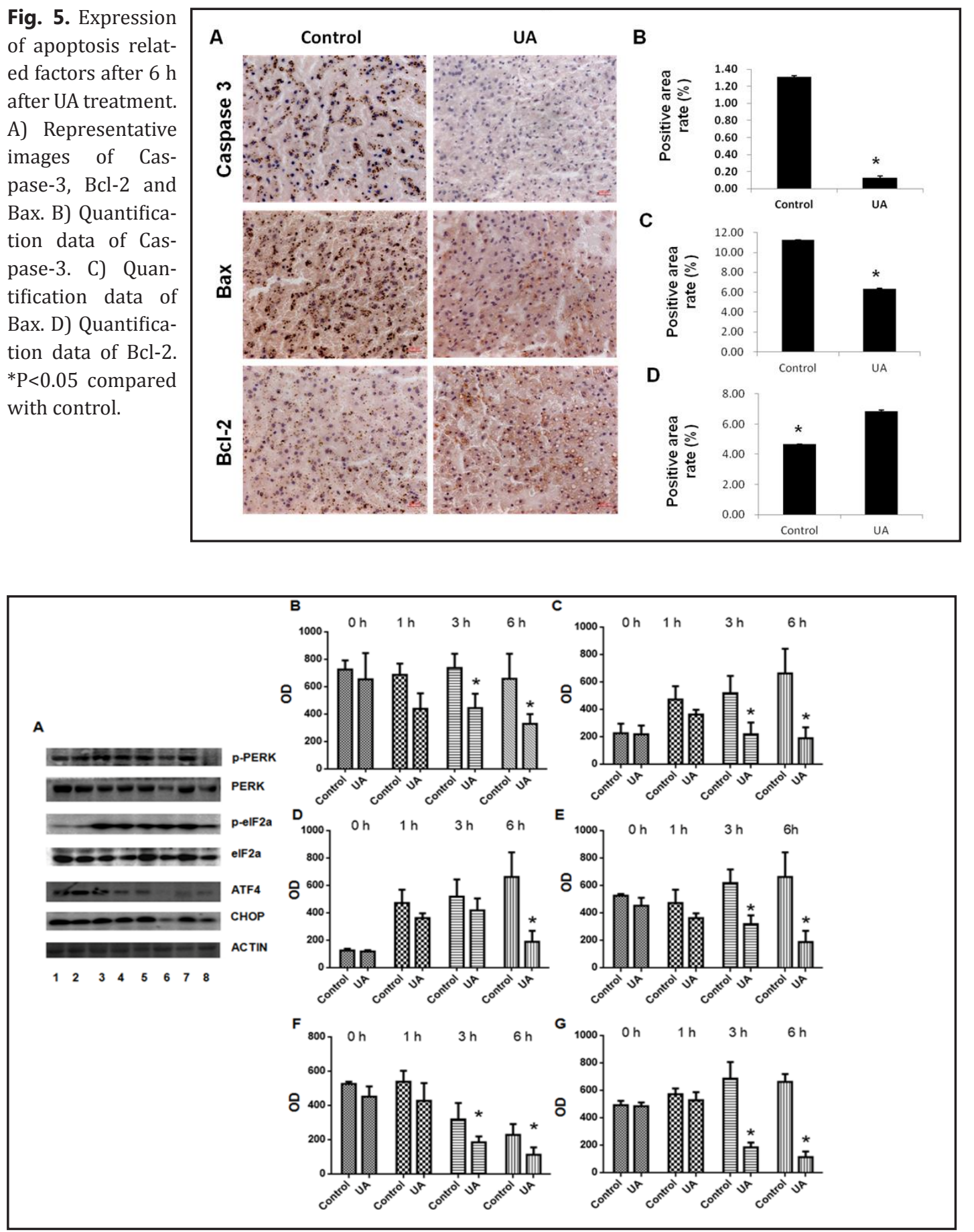

Fig. 6. Expression changes of PERK-CHOP signaling pathway in two groups. A) Representative blots. 1, 3, 5 and 7 were blots for $0,1 \mathrm{~h}, 3 \mathrm{~h}$ and $6 \mathrm{~h}$ from control group. 2, 4, 6 and 8 were blots for $0,1 \mathrm{~h}, 3 \mathrm{~h}$ and $6 \mathrm{~h}$ from UA group B) Quantitative data for PERK expression; C) Quantitative data for p-PERK expression; D) Quantitative data for p-eIF2 $\alpha$ expression; E) Quantitative data for eIF2 $\alpha$ expression; F) Quantitative data for ATF4 expression; G) Quantitative data for CHOP expression. Data were presented with mean and SD. $* \mathrm{P}<0.05$ compared with control group.

Caspase 3 and Bax were significantly decreased, whereas Bcl-2 was increased $6 \mathrm{~h}$ after transplantation in experimental group.

\section{KARGER}




\section{Cellular Physiology Cell Physiol Biochem 2017;43:331-338

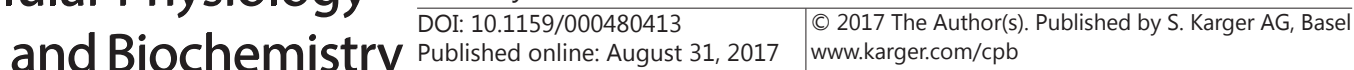 \\ Zhou et al.: UA Treatment Improves the DCD Liver Transplantation}

\section{UA weakened PERK-CHOP pathway}

We also detected the PERK-CHOP signaling pathway by western blotting. As shown in Fig. 6, UA treatment deceased PERK, p-PERK, p-eIF2 $\alpha$, eIF2 $\alpha$, ATF4 and CHOP expression $1 \mathrm{~h}$, $3 \mathrm{~h}$ and $6 \mathrm{~h}$ after transplantation, whereas significant difference was observed at $3 \mathrm{~h}$ and $6 \mathrm{~h}$ regarding p-PERK, PERK, eIF2 $\alpha$, ATF 4 and CHOP. These results suggested that UA treatment decreased PERK-CHOP signaling pathway.

\section{Discussion}

DCD is the main source of donor organs in the field of organ transplantation. Because of the special characteristics in the process of DCD liver transplantation, the time of ischemia and hypoxia is prolonged, and the ischemia reperfusion injury is severe [5, 6]_ENREF_6_ ENREF_1. It is of particular importance to find the mechanism of the liver injury during the process of ischemia and reperfusion and to search relevant interventions to reduce those injuries. In our study, we reported that UA treatment could attenuate DCD liver transplantation-related liver injury. Moreover, serum ALT level and liver tissue MDA level decreased in UA treated group. In our study, we only detected the 24-h pathological changes after transplantation. Although the long-term effect of UA was not evaluated, our study provided positive implication for future study of drug preconditioning in facilitating liver transplantation, especially for the DCD liver transplantation.

Accumulating studies have implicated that liver cell apoptosis is an important form of cell death in both ischemia period or during reperfusion after liver transplantation_ENREF_1. In this study, we disclosed that apoptosis was the major mechanism contributing to the liver injury after transplantation [21-23]. Most importantly, UA pretreatment could alleviate the apoptosis [24]. There are a lot of studies confirmed the role of ERS in ischemia reperfusion injury after liver transplantation [25, 26]_ENREF_22_ENREF_1. Ischemia reperfusion injury leads to a significant increase of liver enzymes, pathological changes and the imbalance of oxidation of glutathione and pathological consumption [27]_ENREF_1. In our study, we evidenced that apoptosis appeared 3-h and aggravated 6-h after transplantation. In addition, we also detected the expressions of apoptosis-related regulators. Caspase- 3 is an apoptosis effector and the high level of caspase-3 indicates the increased apoptosis [24, 28]_ENREF_1. Although we also observed obvious apoptosis in UA treated group, this drug could increase the resistance of the transplanted livers. In our study, we also found significant expression changes of Bax and Bcl-2. UA treatment obviously decreased Bax and increased Bcl-2 expression. In this study, we verified that UA treatment in the experimental group decreased the expressions of various molecules of endoplasmic reticulum stress, including PERK, eIF2 $\alpha$, ATF4 and CHOP within 6 hours after transplantation. Although Bax and Bcl-2 were also altered in UA treated group, it did not exclude the endoplasmic reticulum stress involved in the apoptosis.

UA is a type of compound with the structure of pentacyclic triterpenes. The pharmacological activities have been extensively studied, including antioxidant, antitumor, anti-aging, liver protection etc [29, 30]._ENREF_26_ENREF_1. Because antioxidant has been verified in the protection of organ ischemia reperfusion [19]_ENREF_1, we selected UA in this study and demonstrated that UA likely functioned through decreasing ER stress-related apoptosis. Nevertheless, the reason why UA down-regulates PERK-CHOP pathway is still open. In addition, UA possesses anti-inflammation effects, as well as promotes inflammation and causes DNA injury [31]. Although we did not evaluate the potential toxic effect of UA, it deserves more investigations.

\section{Conclusion}

We reported a novel approach which can ameliorate the DCD liver transplantation related injury. UA likely decreases liver tissue injury through decreasing PERK-CHOP 


\section{Cellular Physiology Cell Physiol Biochem 2017;43:331-338 \begin{tabular}{l|l} 
DOI: 10.1159/000480413 & and Biochemistry \\
Published online: August 31, 2017 & $\begin{array}{l}\text { O 2017 The Author(s). Published by S. Karger AG, Basel } \\
\text { www.karger.com/cpb }\end{array}$
\end{tabular} \\ Zhou et al.: UA Treatment Improves the DCD Liver Transplantation}

signaling pathway mediated apoptosis. These data implicate that UA might have clinical efficacy to improve DCD liver transplantation.

\section{Abbreviations}

UA (ursolic acid); DCD (donation after cardiac death); MDA (malondialdehyde); PERK ( protein kinase-like ER kinase); ALT (aminotransferase); IRI (ischemia/reperfusion injury); ERS (endoplasmic reticulum stress); UPR (unfolded protein response); IRE1 $\alpha$ (inositolrequiring enzyme 1 $\alpha$ ); ATF6 (activating transcription factor 6);

\section{Acknowledgements}

This study is financed by Ministry of health industry fund (201302009, 201002004).

\section{Disclosure Statement}

None.

\section{References}

$\checkmark 1$ Chae MS, Koo JM, Park CS: Predictive Role of Intraoperative Serum Brain Natriuretic Peptide for Early Allograft Dysfunction in Living Donor Liver Transplantation. Ann Transplant 2016;21:538-549.

-2 Dabbous H, Sakr M, Abdelhakam S, Montasser I, Bahaa M, Said H, El-Meteini M: Living donor liver transplantation for high model for end-stage liver disease score: What have we learned? World J Hepatol 2016;8:924-948.

3 Alhamad T, Spatz C, Uemura T, Lehman E, Farooq U: The outcomes of simultaneous liver and kidney transplantation using donation after cardiac death organs. Transplantation 2014;98:1190-1198.

4 Wadei HM, Taner CB: Simultaneous liver-kidney transplantation from donation after cardiac death donors: is there a better way to use these organs? Transplantation 2015;99:e39.

5 Dutkowski P, Polak WG, Muiesan P, Schlegel A, Verhoeven CJ, Scalera I, DeOliveira ML, Kron P, Clavien PA: First Comparison of Hypothermic Oxygenated PErfusion Versus Static Cold Storage of Human Donation After Cardiac Death Liver Transplants: An International-matched Case Analysis. Ann Surg 2015;262:764770.

6 Liu Q, Nassar A, Farias K, Buccini L, Mangino MJ, Baldwin W, Bennett A, O’Rourke C, Iuppa G, Soliman BG, Urcuyo-Llanes D, Okamoto T, Uso TD, Fung J, Abu-Elmagd K, Miller C, Quintini C: Comparing Normothermic Machine Perfusion Preservation With Different Perfusates on Porcine Livers From Donors After Circulatory Death. Am J Transplant 2016;16:794-807.

7 Luo Y, Ji WB, Duan WD, Ye S, Dong JH. Graft cholangiopathy: etiology, diagnosis, and therapeutic strategies. Hepatobiliary Pancreat Dis Int 2014;13:10-17.

8 Kageyama S, Yagi S, Tanaka H, Saito S, Nagai K, Hata K, Fujimoto Y, Ogura Y, Tolba R, Shinji U. Graft reconditioning with nitric oxide gas in rat liver transplantation from cardiac death donors. Transplantation 2014;97:618-625.

9 Vilatoba M, Eckstein C, Bilbao G, Smyth CA, Jenkins S, Thompson JA, Eckhoff DE, Contreras JL: Sodium 4-phenylbutyrate protects against liver ischemia reperfusion injury by inhibition of endoplasmic reticulum-stress mediated apoptosis. Surgery 2005;138:342-351.

- 10 Lu H, Lu L, Xu ZC, Lu YJ, Zhao B, Zhuang L, Hao BB, Zhang F: Tauroursodeoxycholic acid and 4-phenyl butyric acid alleviate endoplasmic reticulum stress and improve prognosis of donation after cardiac death liver transplantation in rats. Hepatobiliary Pancreat Dis Int 2014;13:586-593.

11 Finka A, Sharma SK, Goloubinoff P: Multi-layered molecular mechanisms of polypeptide holding, unfolding and disaggregation by HSP70/HSP110 chaperones. Front Mol Biosci 2015;2:29. 


\section{Cellular Physiology Cell Physiol Biochem 2017;43:331-338 \begin{tabular}{ll|l} 
DOI: 10.1159/000480413 & and Biochemistry & $\begin{array}{l}\text { O } 2017 \text { The Author(s). Published by S. Karger AG, Basel } \\
\text { wwww.karger.com/cpb }\end{array}$
\end{tabular}

12 Wu J, Ruas JL, Estall JL, Rasbach KA, Choi JH, Ye L, Boström P, Tyra HM, Crawford RW, Campbell KP, Rutkowski DT, Kaufman RJ, Spiegelman BM: The unfolded protein response mediates adaptation to exercise in skeletal muscle through a PGC-1alpha/ATF6alpha complex. Cell Metab 2011;13:160-169.

-13 Oshitari T, Yoshida-Hata N, Yamamoto S: Effect of neurotrophin-4 on endoplasmic reticulum stress-related neuronal apoptosis in diabetic and high glucose exposed rat retinas. Neurosci Lett 2011;501:102-106.

14 Shi YH, Ding ZB, Zhou J, Hui B, Shi GM, Ke AW, Wang XY, Dai Z, Peng YF, Gu CY, Qiu SJ, Fan J: Targeting autophagy enhances sorafenib lethality for hepatocellular carcinoma via ER stress-related apoptosis. Autophagy 2011;7:1159-1172.

15 Yang Y, Li C, Xiang X, Dai Z, Chang J, Zhang M, Cai H, Zhang H, Zhang M, Guo Y, Wu Z: Ursolic acid prevents endoplasmic reticulum stress-mediated apoptosis induced by heat stress in mouse cardiac myocytes. J Mol Cell Cardiol 2014;67:103-111.

16 Li JS, Wang WJ, Sun Y, Zhang YH, Zheng L: Ursolic acid inhibits the development of nonalcoholic fatty liver disease by attenuating endoplasmic reticulum stress. Food Funct 2015;6:1643-1651.

17 Minor T, Koetting M, Koetting M, Kaiser G, Efferz P, Lüer B, Paul A: Hypothermic reconditioning by gaseous oxygen improves survival after liver transplantation in the pig. Am J Transplant 2011;11:2627-2634.

18 Shao Z, Jiao B, Liu T, Cheng Y, Liu H, Liu Y: TAK-242 treatment ameliorates liver ischemia/reperfusion injury by inhibiting TLR4 signaling pathway in a swine model of Maastricht-category-III cardiac death. Biomed Pharmacother 2016;84:495-501.

19 Li L, Zhang X, Cui L, Wang L, Liu H, Ji H, Du Y: Ursolic acid promotes the neuroprotection by activating Nrf2 pathway after cerebral ischemia in mice. Brain Res 2013;1497:32-39.

20 Zhu G, Liu Y, Wang Y, Bi X, Baudry M: Different patterns of electrical activity lead to long-term potentiation by activating different intracellular pathways. J Neurosci 2015;35:621-633.

-21 Li B, Chen B, Zhang G, Wang K, Zhou L, Hu S: Cell apoptosis and Fas gene expression in liver and renal tissues after ischemia-reperfusion injury in liver transplantation. Transplant Proc 2010;42:1550-1556.

-22 Yang H, Zhou H, Zhuang L, Wang X, Lv L: GPBAR1/TGR5 Attenuates Liver Ischemia/Reperfusion Injury via the Inhibition of TLR4 Signaling in Mice. Liver Transpl 2017;23:63-74.

23 Li H, Sun JJ, Chen GY, Wang WW, Xie ZT, Tang GF, Wei SD: Carnosic acid nanoparticles suppress liver ischemia/reperfusion injury by inhibition of ROS, Caspases and NF-kappaB signaling pathway in mice. Biomed Pharmacother 2016;82:237-246.

24 Xu Y, Guo M, Jiang W, Dong H, Han Y, An XF, Zhang J: Endoplasmic reticulum stress and its effects on renal tubular cells apoptosis in ischemic acute kidney injury. Ren Fail 2016;38:831-837.

-25 Zhou H, Zhu J, Yue S, Lu L, Busuttil RW, Kupiec-Weglinski JW, Wang X, Zhai Y: The Dichotomy of Endoplasmic Reticulum Stress Response in Liver Ischemia-Reperfusion Injury. Transplantation 2016;100:365-372.

-26 Folch-Puy E, Panisello A, Oliva J, Lopez A, Castro Benítez C, Adam R, Roselló-Catafau J: Relevance of Endoplasmic Reticulum Stress Cell Signaling in Liver Cold Ischemia Reperfusion Injury. Int J Mol Sci doi:10.3390/ijms17060807.

-27 Geng W, Lo CM, Ng KT, Ling CC, Qi X, Li CX, Zhai Y, Liu XB, Ma YY, Man K: Interferon-gamma inducible protein 10 (IP10) induced cisplatin resistance of HCC after liver transplantation through ER stress signaling pathway. Oncotarget 2015;6:28042-28056.

-28 Zhu G, Wang X, Wu S, Li Q: Involvement of activation of PI3K/Akt pathway in the protective effects of puerarin against MPP+-induced human neuroblastoma SH-SY5Y cell death. Neurochem Int 2012;60:400408.

-29 Smethurst DG, Cooper KF: ER fatalities-The role of ER-mitochondrial contact sites in yeast life and death decisions. Mech Ageing Dev 2017;161:225-233.

-30 Li D, Ren D, Luo Y, Yang X: Protective effects of ursolic acid against hepatotoxicity and endothelial dysfunction in mice with chronic high choline diet consumption. Chem Biol Interact 2016;258:102-107.

-31 Peng J, Ren X, Lan T, Chen Y, Shao Z, Yang C: Renoprotective effects of ursolic acid on ischemia/ reperfusioninduced acute kidney injury through oxidative stress, inflammation and the inhibition of STAT3 and NFkappaB activities. Mol Med Report 2016;14:3397-402. 